\title{
Dietary protein ingested before and during short photoperiods makes an impact on affect-related behaviours and plasma composition of amino acids in mice
}

\author{
Tsuyoshi Otsuka†, Ryosei Goda†, Ayaka Iwamoto, Misato Kawai, Satomi Shibata, Yoshiaki Oka, \\ Wataru Mizunoya, Mitsuhiro Furuse and Shinobu Yasuo* \\ Faculty of Agriculture, Kyushu University, Hakozaki 6-10-1, Higashi-ku, Fukuoka 812-8581, Japan
}

(Submitted 27 April 2015 - Final revision received 18 June 2015 - Accepted 4 August 2015 - First published online 15 September 2015)

\begin{abstract}
In mammals, short photoperiod is associated with high depression- and anxiety-like behaviours with low levels of the brain serotonin and its precursor tryptophan (Trp). Because the brain Trp levels are regulated by its ratio to large neutral amino acids (Trp:LNAA) in circulation, this study elucidated whether diets of various protein sources that contain different Trp:LNAA affect depression- and anxiety-like behaviours in C57BL/6J mice under short-day conditions (SD). In the control mice on a casein diet, time spent in the central area in the open field test (OFT) was lower in the mice under SD than in those under long-day conditions (LD), indicating that SD exposure induces anxiety-like behaviour. The SD-induced anxiety-like behaviour was countered by an $\alpha$-lactalbumin diet given under SD. In the mice that were on a gluten diet before transition to SD, the time spent in the central area in the OFT under SD was higher than that in the SD control mice. Alternatively, mice that ingested soya protein before the transition to SD had lower immobility in the forced swim test, a depression-like behaviour, compared with the SD control. Analysis of Trp:LNAA revealed lower Trp:LNAA in the SD control compared with the LD control, which was counteracted by an $\alpha$-lactalbumin diet under SD. Furthermore, mice on gluten or soya protein diets before transition to SD exhibited high Trp:LNAA levels in plasma under SD. In conclusion, ingestion of specific proteins at different times relative to photoperiodic transition may modulate anxietyand/or depression-like behaviours, partially through changes in plasma Trp:LNAA.
\end{abstract}

Key words: $\boldsymbol{\alpha}$-Lactalbumin: Gluten: Photoperiodism: Seasonal affective disorder: Soya protein

In mammals, photoperiod regulates various physiological functions and behaviours, including breeding, metabolism and stress- and affect-related behaviours ${ }^{(1-6)}$. In humans, seasonal affective disorder (SAD) is characterised by the occurrence of depression, hypersomnia, hyperphagia and carbohydrate craving during specific seasons, usually winter ${ }^{(7)}$. Pathogenesis of SAD is known to involve seasonal fluctuations in the brain serotonergic system. First, serotonin (5-HT) levels in the postmortem hypothalamic tissue from human subjects were lower in the tissue collected in winter compared with the tissue collected in summer ${ }^{(8)}$. Second, in healthy men, estimated 5-HT turnover in the brain was lowest in winter and correlated with the number of hours of bright sunlight ${ }^{(9)}$. Third, plasma levels of tryptophan (Trp), a precursor of 5-HT, fluctuated with seasons in healthy volunteers ${ }^{(10)}$. Finally, 5-HT transporter function during depression is enhanced in patients with SAD; it normalises after light therapy and in natural summer remission ${ }^{(11)}$.

5-HT levels in the brain depend on the amount of Trp transported from the peripheral circulation into the brain through the blood-brain barrier ${ }^{(12)}$. Trp is one of the large neutral amino acids (LNAA, which include Trp, tyrosine, phenylalanine, valine, leucine and isoleucine), all of which compete for the same transporter system in the blood-brain barrier $^{(13)}$. Thus, Trp levels in the brain are correlated with ratios of Trp levels to levels of other LNAA (Trp:LNAA) in circulation; high Trp:LNAA result in high Trp levels in the brain $^{(12)}$. Trp:LNAA are regulated by dietary carbohydrate intake, which is associated with insulin secretion in both animals and humans ${ }^{(14,15)}$. Insulin promotes the uptake of branched-chain amino acids (valine, leucine and isoleucine) into the skeletal muscle; consequently, Trp:LNAA increase in the plasma because of the decrease in these competing amino acids. On the basis of these mechanisms, carbohydrate craving in the SAD patients, which is a typical symptom of SAD, is hypothesised to be a form of self-medication to compensate for the reduction of 5-HT levels in the brain. In accordance with this hypothesis, SAD patients frequently report an activating effect of carbohydrates ${ }^{(16)}$, and a Trp-deficient diet causes a

Abbreviations: 5-HT, serotonin; FST, forced swim test; LD, long-day conditions; LNAA, large neutral amino acids; OFT, open field test; SAD, seasonal affective disorder; SD, short-day conditions; Trp, tryptophan.

* Corresponding author: S. Yasuo, fax +81 92642 4426, email syasuo@brs.kyushu-u.ac.jp

$\dagger$ Both authors contributed equally to this work. 
relapse of depression ${ }^{(17)}$. Seasonal variation of plasma Trp: LNAA was also reported in healthy volunteers ${ }^{(10)}$.

Several animals have been proposed as animal models of SAD, including fat sand rats ${ }^{(1)}$, grass rats $^{(3)}$ and Siberian hamsters $^{(6)}$. As laboratory mouse strains including $\mathrm{C} 57 \mathrm{BL} / 6 \mathrm{~J}$ cannot produce detectable levels of melatonin, which is an important photoperiodic messenger, they were thought to be an inappropriate model for SAD. Recently, however, we found that C57BL/6J mice under short-day condition (SD) exhibited amplified plasma corticosterone rhythms, whereas those maintained under long-day conditions (LD) exhibited no significant rhythmicity $^{(4)}$. In addition, C57BL/6J mice under SD, compared with mice under LD, showed high immobility in the forced swim test (FST) and a low preference for saccharin, which are considered depression-like behaviours. Furthermore, these mice showed high sucrose preference, which was accompanied by low levels of 5-HT in the brain and low Trp: LNAA in plasma ${ }^{(5)}$. Thus, these mice may mirror depressive symptoms and carbohydrate cravings with neurochemical changes in SAD patients.

In this study, we elucidated whether modification of plasma Trp:LNAA by dietary protein sources (casein, soya protein, gluten and $\alpha$-lactalbumin) that differ in Trp:LNAA affects depression- and anxiety-like behaviours in the C57BL/6J mice under SD. Previous studies showed that acute and chronic intakes of the diet with these proteins significantly altered plasma Trp:LNAA, brain Trp levels and 5-HT synthesis in rats $^{(18-20)}$. We also examined whether the intake of these diets before the transition to SD prevents the SD-induced anxietyand depression-like behaviours. As plasma Trp:LNAA are strongly associated with metabolic activity of fat and skeletal muscle, we also analysed epididymal fat mass, plasma concentration of NEFA and muscle fibre types (I, slow-twitch type; IIa and IIx, intermediate type; IIb, fast-twitch type) that have different roles in metabolism ${ }^{(21)}$

\section{Methods}

\section{Animals}

Male C57BL/6J mice (4 weeks old) were purchased from Japan SLC and randomly housed in plastic cages in groups of three. All mice were housed in boxes placed in a room at $25 \pm 1^{\circ} \mathrm{C}$ with LD (18 h of light (50 lux) and $6 \mathrm{~h}$ of darkness (18L6D)) for at least 1 week before the experiment. The control diet (described below) and tap water were provided ad libitum. All animal experiments were conducted in accordance with the Guidelines for Animal Experiments of the Faculty of Agriculture at Kyushu University, as well as the Law (No. 105) and Notification (No. 6) of the Japanese Government.

\section{Diets}

The composition of experimental diets is shown in Table 1. All diets contained $170 \mathrm{~g}$ of crude protein $/ 1 \mathrm{~kg}$ diet. The percentage of crude protein in casein, gluten, soya protein or $\alpha$-lactalbumin powder varies $(85,90,83$ and $94 \%$, respectively); therefore, the amount of the casein, gluten, soya protein or $\alpha$-lactalbumin
Table 1. The composition of experimental diets ( $\mathrm{g} / \mathrm{kg}$ diet)

\begin{tabular}{lcclc}
\hline & Casein & Soya protein & Gluten & $a$-Lactalbumin \\
\hline Protein powder & $200^{*}$ & $189^{*}$ & $205^{*}$ & $181^{*}$ \\
Maize starch & 529.486 & $540 \cdot 486$ & $525 \cdot 886$ & 550.586 \\
Sucrose & 100 & 100 & 100 & 100 \\
Soyabean oil & 70 & 70 & 70 & 70 \\
Cellulose & 50 & 50 & 50 & 50 \\
Mineral mix $\dagger$ & 35 & 35 & 35 & 35 \\
Vitamin mix $\dagger$ & 10 & 10 & 10 & 10 \\
L-Cys & 3 & 3 & - & - \\
L-Lys & - & - & 1.6 & - \\
L-Arg & - & - & - & 0.9 \\
Choline bitartrate & 2.5 & 2.5 & 2.5 & 2.5 \\
Butylhydroquinone & 0.014 & 0.014 & 0.014 & 0.014 \\
\hline
\end{tabular}

* Crude protein levels are identical to $170 \mathrm{~g} / \mathrm{kg}$ diet in all diets.

$\dagger$ The vitamin and mineral mixes were purchased from KBT Oriental and contain 97 and $22 \%$ sucrose, respectively.

Table 2. Tryptophan and related amino acid compositions in protein powders

\begin{tabular}{lcccc}
\hline & Casein & Soya protein & Gluten & $a$-Lactalbumin \\
\hline Trp $(\mathrm{g} / \mathrm{kg})$ & 11 & 14 & 7.9 & 44.3 \\
BCAA $(\mathrm{g} / \mathrm{kg})$ & 190 & 168 & 117.2 & 213 \\
Trp:LNAA & $0.0388^{\star}$ & $0.0543^{\star}$ & $0.0405^{\star}$ & $0.1287^{\star}$ \\
\hline
\end{tabular}

BCAA, branched-chain amino acids; LNAA, large neutral amino acids.

* The ratio was calculated on the basis of the amino acid compositions analysed by Japan Food Research Laboratories (casein, gluten and $a$-lactalbumin) or Fuji Oil Co., Ltd (soya protein).

powder used was 200, 189, 205 and $181 \mathrm{~g} / \mathrm{kg}$ diet, respectively. The amount was balanced with maize starch. On the basis of the nutrient requirements for rodents, diets with casein and soya proteins were supplemented with L-cystine. The gluten diet was supplemented with L-lysine, and the $\alpha$-lactalbumin diet was supplemented with L-arginine. All diets were prepared in our laboratory using ingredients shown in Table 1. All diets were mixed with methylcellulose solution (0.25\%) and dried in an air-dried oven to form a pellet. The casein diet was used as the control diet. Trp and branched-chain amino acid levels and Trp:LNAA in the experimental diets are shown in Table 2.

\section{Experimental design}

After acclimation, cages of mice were divided into eight groups (three cages with nine animals per group). Mice were group housed, because our preliminary data revealed that social isolation of $\mathrm{C} 57 \mathrm{BL} / 6 \mathrm{~J}$ mice masked the effect of photoperiod on immobility in the FST (Y Togo, T Otsuka, M Furuse and $S$ Yasuo, unpublished results). The first and second groups were assigned as the SD control and LD control groups, respectively (Fig. 1). SD control mice were exposed to LD for the first 3 weeks and then transferred to SD (6L18D) with the casein diet for the rest of the experimental period. The LD control mice were maintained on a casein diet throughout the experimental period. The third to fifth groups were exposed to LD for 3 weeks with the casein diet, and the lighting condition was then changed to SD (6L18D) with replacement of the casein diet with one of gluten, soya protein or $\alpha$-lactalbumin (Fig. 1). 


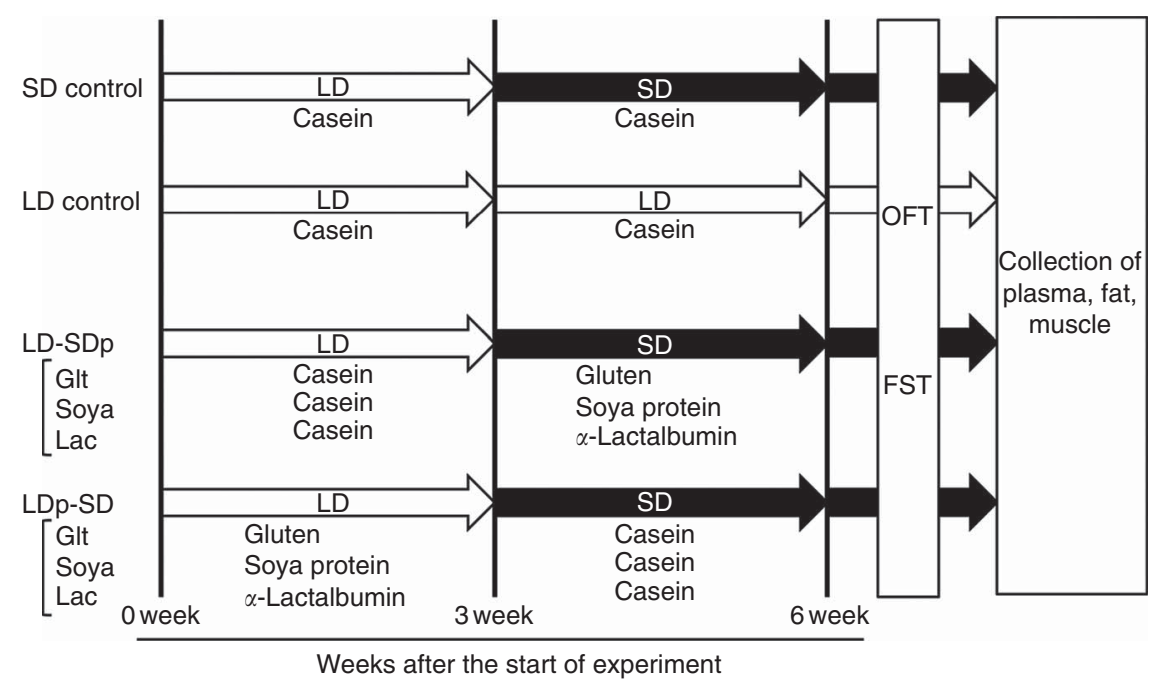

Fig. 1. Experimental procedure. Short-day condition (SD) control mice were housed under long-day condition (LD) (18h of light and $6 \mathrm{~h}$ of darkness: 18L6D) for the first 3 weeks, followed by exposure to SD (6L18D) and a casein diet. LD control mice were housed under LD throughout the experiment and fed a casein diet. The LD-SDprotein (SDp) group was first housed under LD for 3 weeks while on a casein diet, followed by exposure to SD while on gluten, soya protein or $a$-lactalbumin diets. The LDprotein (LDp)-SD groups were first housed under LD for 3 weeks while on either gluten, soya protein or a-lactalbumin diets, followed by exposure to SD while on a casein diet. After 6 weeks of the start of the experiment, an open field test (OFT) and forced swim test (FST) were performed. Plasma samples and skeletal muscle were collected for analysis of plasma (tryptophan:large neutral amino acids) and muscle fibre type 4-5d after the FST. Glt, gluten; soya, soya protein; lac, $\alpha$ lactalbumin).

These groups were considered the LD-SDprotein (SDp) groups. The sixth to eighth groups were exposed to LD for 3 weeks while on a gluten, soya protein or $\alpha$-lactalbumin diet, and they were then exposed to SD while on a casein diet (Fig. 1). These groups were considered the LDprotein (LDp)-SD groups. Food intake (as a cage) in all groups was measured every week, and the total during weeks 2-3 and 5-6 after the start of the experiment was calculated for analysis. After 3 weeks of the last switch of the light/dietary conditions, open field test (OFT) and FST were performed at 2-d intervals. Photoperiod and dietary conditions were continued during these behavioural tests. Mice were euthanised 3, 9 or $21 \mathrm{~h}$ after the lights-on (three animals per time point per group) with isoflurane $4-5 \mathrm{~d}$ after the FST, and plasma for analysis of Trp:LNAA and gastrocnemius for muscle fibre type analysis were collected. Body weight and epididymal fat mass were measured on the day of euthanisation. Because there were no significant effects of time of day on the parameters examined, the data of three time points were mixed for further analysis. All experimental groups (SD and LD controls, LD-SDp and LDp-SD) were run together in the same trial.

\section{Behavioural tests}

OFT and FST were performed to address the effect of the experimental diets on mood-related behaviours. The tests were performed during light phase under 5 lux light, starting $2 \mathrm{~h}$ after the lights-on, because mood-related behaviours and the brain serotonergic system is highly sensitive to photoperiod during light phase in mice ${ }^{(5)}$. Tests in all mice in all groups were completed within $2 \mathrm{~h}$. The OFT was used to analyse spontaneous activity and anxiety-like behaviour in a novel environment. The use of the FST was advocated by a previous study ${ }^{(22)}$, and it is widely used to analyse behavioural despair or depression-like behaviour.
The OFT was performed in the white square field $(40 \times 40 \mathrm{~cm})$ with $40-\mathrm{cm}$ high walls. Each mouse was placed in the centre of the apparatus and recorded using digital video for 5 min. Open field behaviour was analysed using ANY-maze software (Stoelting Co.) by dividing the field into 25 squares $(5 \times 5$ grid). The number of grid lines that were crossed was used as the parameter of spontaneous activity, and the time spent in the central nine grids was used as the parameter of anxiety-like behaviour.

In the FST, mice were individually put into plastic, transparent cylinders (27-cm high, $17-\mathrm{cm}$ diameter) containing $14 \cdot 5-\mathrm{cm}$-high water at $25 \pm 1^{\circ} \mathrm{C}$. The behaviours were recorded using digital video for $7 \mathrm{~min}$, and immobility time during the last $5 \mathrm{~min}$ of the $7 \mathrm{~min}$ was blindly analysed. The mouse was considered immobile when it floated with its head above the water without moving its tail.

\section{Measurements of plasma NEFA and amino acids}

Blood samples were obtained by decapitation, and plasma was collected after centrifugation at $3000 \mathrm{~g}$ for $10 \mathrm{~min}$ at $4^{\circ} \mathrm{C}$. The plasma samples were stored at $-80^{\circ} \mathrm{C}$ until they were analysed. Plasma NEFA levels were measured using the NEFA C-test kit WAKO (Wako Pure Chemical Industries Ltd) according to the manufacturers' protocol. Free amino acids in the plasma were determined by HPLC (Pico-tag ${ }^{\mathrm{TM}}$; Waters) according to a previous method $^{(23)}$. The following was added to the samples: $1 \mathrm{~m}$-sodium hydroxide, calibrated to $\mathrm{pH} 7 \cdot 0$, and a mixed solution that consisted of $40 \% 1 \mathrm{~m}$-sodium acetate, $40 \%$ methanol and $20 \%$ triethylamine. After drying, these samples were allowed to derivatise with a reaction solution that consisted of $70 \%$ methanol, $10 \%$ water, $10 \%$ triethylamine and $10 \%$ phenylisothiocyanate. The samples were dried again, dissolved in $200 \mu \mathrm{l}$ of Pico-tag sample diluent and filtered 
through a Syringe Driven Filter Unit (Millex-LG; Millipore) to remove the solid contents. A standard amino acid mixture (Type AN II and type B; Wako Pure Chemical Industries Ltd) was applied using the same methods. These derivatised samples and standards were applied to the HPLC system and equilibrated with buffer A (70 mm-sodium acetate, acetonitrile (975:25)) and eluted with buffer B (water, acetonitrile, methanol $(40: 45: 15))$ at a flow rate of $1 \mathrm{ml} / \mathrm{min}$ at $46^{\circ} \mathrm{C}$.

\section{SDS-PAGE}

The gastrocnemius was dissected and quickly frozen with liquid $\mathrm{N}_{2}$. Muscle fibre types were analysed by SDS-PAGE of myosin heavy-chain isoforms, IIa, IIb and IIx, according to the previous report $^{(24)}$. Muscle samples were homogenised in a solution containing $10 \% \mathrm{w} / \mathrm{v}$ SDS, $40 \mathrm{~mm}$-dithiothreitol, $5 \mathrm{~mm}$-EDTA and $0 \cdot 1 \mathrm{~m}$-Tris- $\mathrm{HCl}$ buffer. The separating gel consisted of $35 \% \mathrm{v} / \mathrm{v}$ glycerol, $\quad 8 \% \quad \mathrm{w} / \mathrm{v}$ acrylamide- $N, N$-methylenebisacrylamide (99:1), 0.2 м-Tris- $\mathrm{HCl}(\mathrm{pH} 8.8), 0.1 \mathrm{~m}$ glycine, $0.4 \% \mathrm{w} / \mathrm{v}$ SDS, $0.1 \% \mathrm{w} / \mathrm{v}$ ammonium persulfate and $0.05 \% \mathrm{v} / \mathrm{v} \quad N, N, N$, $N^{\prime}$-tetramethylethylenediamine. Samples containing $100 \mathrm{ng}$ of protein were applied to the gel and electrophoresed at a constant voltage of $140 \mathrm{~V}$ for $23 \mathrm{~h}$ except for the first $40 \mathrm{~min}$, where the maximum current was limited to $10 \mathrm{~mA}$. The gel unit was placed at $4^{\circ} \mathrm{C}$ throughout the electrophoresis. After the run, gels were stained using a silver stain kit (Silver Stain KANTO III; Kanto Chemicals) and dried using a Gel Dry System (Tefco). These methods have been shown to separate myosin heavy-chain isoforms in a specific manner, as confirmed by Western blotting using antibodies against each isoform ${ }^{(24)}$. We could not clearly separate the bands of IIa and IIx, and thus the bands of IIb and IIa + IIx were quantified densitometrically using the ImageJ 1.47 software (National Institutes of Health). The data are expressed as percentage of IIb content (fast-twitch fibre) to the sum of IIa, IIb and IIx contents.

\section{Statistical analysis}

LD-SDp and LDp-SD groups were analysed separately. SD and LD controls were used for the control for both the LD-SDp and LDp-SD groups. One-way ANOVA was used to analyse the effects of the diets on food intake, body and epididymal fat weights, plasma NEFA concentrations, OFT, FST and Trp:LNAA. When significance $(P<0.05)$ was detected, a post hoc test was performed using Dunnett's multiple comparison test to compare the values in the SD or LD control group. Behavioural parameters and Trp:LNAA were also tested using linear mixed models (SAS, PROC MIXED; SAS Institute) with photoperiod or diet as a fixed factor to evaluate the contribution of these factors. In case of Trp: LNAA, time of day was treated as a random effect.

\section{Results}

Effects of various proteins on food intake, body weight, epididymal fat mass and plasma NEFA concentrations

In the LD-SDp groups, food intake during weeks 5-6 but not weeks $2-3$ differed significantly $(P=0 \cdot 0134)$ between groups.
The mice that were on an $\alpha$-lactalbumin diet showed significantly $(P<0.01)$ lower levels of food intake compared with those in the SD and LD control groups (Fig. 2(a)). In the LDp-SD groups, food intake from weeks 2-3 but not from weeks 5-6 was significantly ( $P=0.0032)$ different between diets (Fig. 2(b)). Post boc analysis revealed that mice fed the gluten, soya protein or $\alpha$-lactalbumin diet ate less food (gluten: $P<0.001$, soya protein and $\alpha$-lactalbumin: $P<0.05$ ) than mice fed the casein diet in the SD control group. Food intake in the mice fed the gluten diet was significantly $(P<0.05)$ lower than that in the LD control group.

Body and epididymal fat weights were higher in the SD control group than in the LD control group (body weight: $P<0 \cdot 01$, fat: $P<0.05)$. In the LD-SDp group, body and epididymal fat weights significantly differed by diet (body weight: $P<0.0001$, fat: $P=0.0012$ ). The mice that were on an $\alpha$-lactalbumin diet exhibited significantly (body weight: $P<0 \cdot 0001$, fat: $P<0 \cdot 001$ ) lower levels of body and epididymal fat weights compared with those in the SD control group (Fig. 2(c) and (e)). Similar results were obtained in the LDp-SD group (body weight: $P=0.0138$, fat: $P=0.0081$ ); both body and epididymal fat weights in the mice that were on an $\alpha$-lactalbumin diet were significantly (body weight: $P<0.05$, fat: $P<0 \cdot 01$ ) lower than those in the SD control group (Fig. 2(d) and (f)). When epididymal fat mass was divided by body weight in each mouse, the mice that were on an $\alpha$-lactalbumin diet in the LD-SDp groups but not in the LDp-SD groups exhibited significantly $(P<0.05)$ lower levels compared with those in the SD control group (Fig. 2(g) and (h)).

Plasma concentrations of NEFA significantly varied in both LD-SDp $(P=0.0142)$ and LDp-SD $(P=0.0102)$ groups (Fig. 2(i) and (j)). Post hoc analysis revealed that the NEFA levels were higher in the SD control group than in the LD control group $(P<0.05$ in LD-SDp, $P<0.01$ in LDp-SD). In the LD-SDp group, mice that were on an $\alpha$-lactalbumin diet exhibited significantly lower levels of NEFA than those in the SD control group $(P<0 \cdot 05)$.

\section{Effects of various proteins on behaviours in the open field} test and forced swim test

In the OFT, the number of grid lines crossed was not affected by photoperiod or diets with various proteins in both the LD-SDp and LDp-SD groups (Fig. 3(a) and (b)). In the LD-SDp groups, the time spent in the centre area is significantly affected by photoperiod $(P=0.0228)$ and $\operatorname{diet}(P=0.0089)$. One-way ANOVA revealed that it differed significantly between groups $(P=0.0085)$; the SD control group exhibited significantly $(P<0.05)$ lower levels than the LD control group (Fig. 3(c)). The SD-induced suppression of the time spent in the centre area was restored by an $\alpha$-lactalbumin diet $(P<0 \cdot 01)$ (Fig. 3(c)). Alternatively, in the LDp-SD groups, significant effect of photoperiod $(P=0.0122)$ and $\operatorname{diet}(P=0.0426)$ was detected. A significant $(P=0.0194)$ variation was detected among groups, and the reduction in time spent in the centre area under SD was prevented by prior ingestion of a gluten diet $(P<0.05)$ (Fig. 3(d)).

In the FST, there was no significant effect of photoperiod or experimental diets on the immobility in the LD-SDp 
(a)

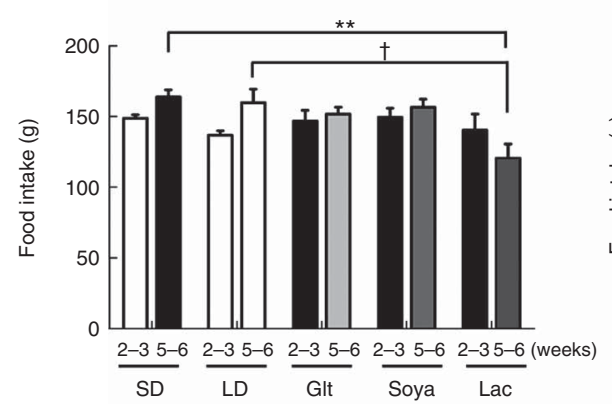

(c)

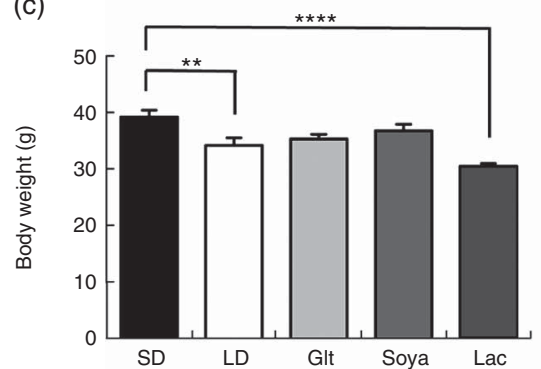

(e)

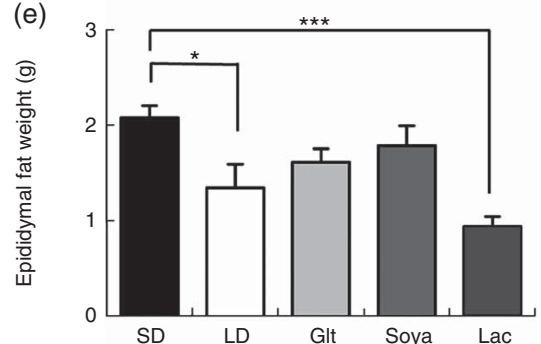

(g)

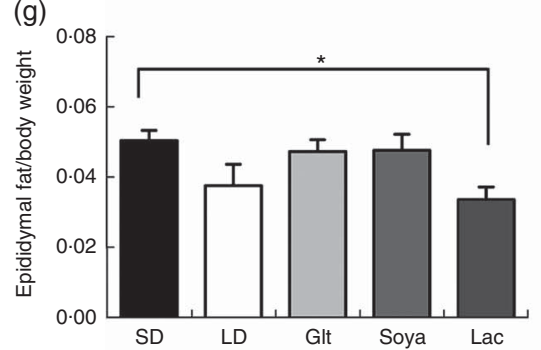

(i)

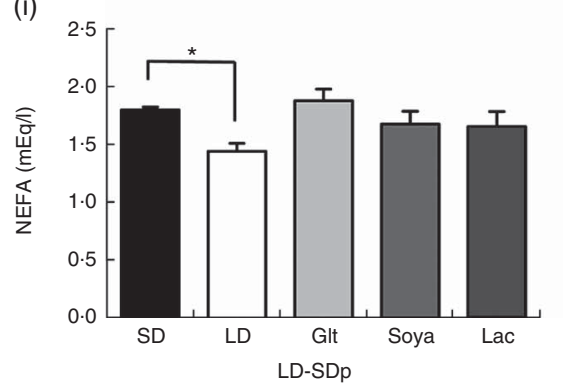

(b)

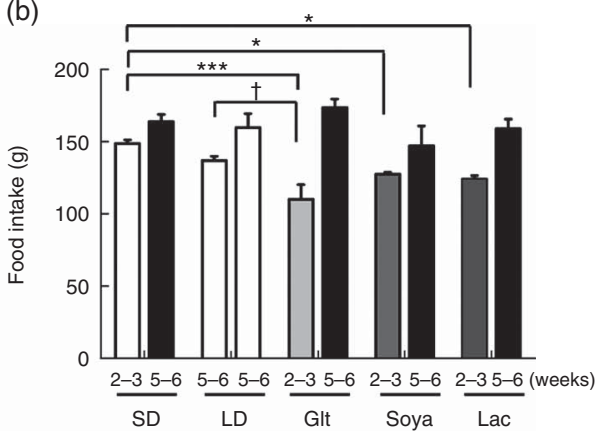

(d)

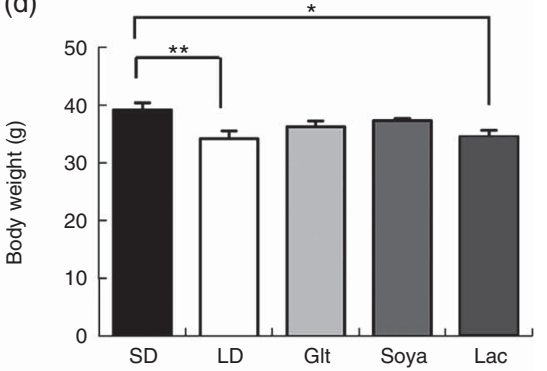

(f)

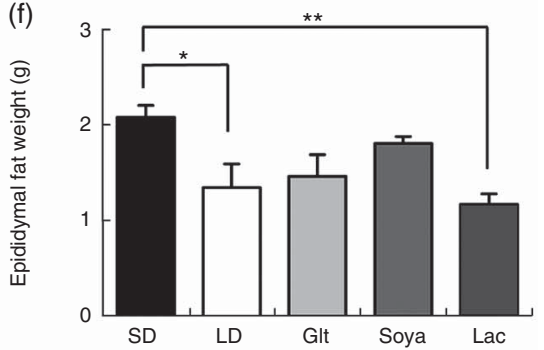

(h)

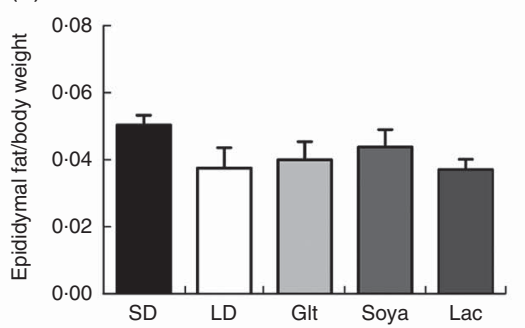

(j)

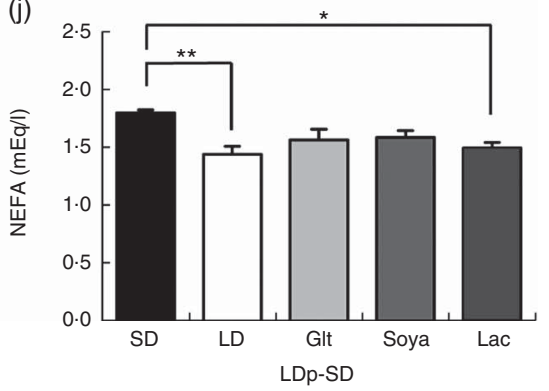

Fig. 2. Effects of various protein diets (glt, gluten; soya, soya protein; lac, a-lactalbumin) on food intake (a, b), body weight (c, d), fat weight (e, f), fat weight divided by body weight $(\mathrm{g}, \mathrm{h})$ and plasma levels of NEFA (i, j) in the long-day condition (LD)-short-day condition (SD) protein (SDp) (a, c, e, $g$, i) and LD protein (LDp)-SD $(b, d, f, h, j)$ groups. The data of the SD and LD controls were depicted in both the LD-SDp and LDp-SD groups. Values are means ( $n 9$ except for food intake, which was measured as a cage (three cages in each group)), with their standard errors are represented by vertical bars. Statistically significant difference: ${ }^{*} P<0.05$, ${ }^{\star *} P<0.01,{ }^{\star \star \star} P<0.001,{ }^{\star \star \star \star} P<0.0001$ (Dunnett's test) v. SD control: $\dagger P<0.05$ (Dunnett's test) $v$. LD control. 

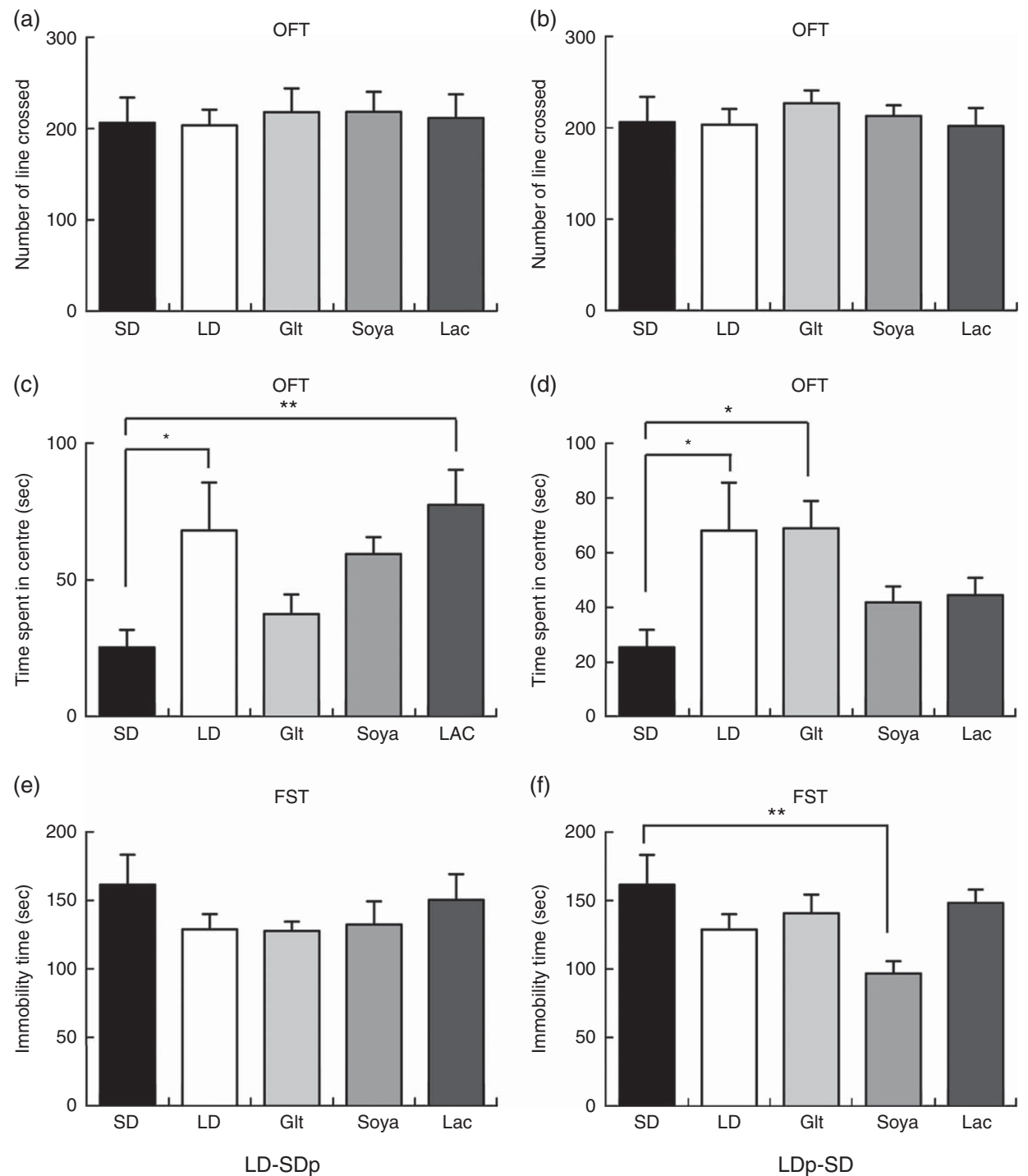

Fig. 3. Effects of various protein diets (glt, gluten; soya, soya protein; lac, $a$-lactalbumin) on behaviours in the open field test (OFT: a, b, c, d) and forced swim test (FST: e, f) in the long-day condition (LD)-short-day condition (SD) protein (SDp) (a, c, e) and LDprotein (LDp)-SD (b, d, f) groups. Values are means ( $n$ 9), with their standard errors are represented by vertical bars. Statistically significant difference: ${ }^{*} P<0.05$, ${ }^{* *} P<0.01$ (Dunnett's test) $v$. SD control.

groups (Fig. 3(e)). In the LDp-SD groups, the experimental diets, but not photoperiod, significantly $(P=0.0176)$ affected immobility. A significant $(P=0 \cdot 0248)$ variation was detected among groups; the mice that were on a soya protein diet exhibited significantly $(P<0 \cdot 01)$ lower immobility compared with the SD control group (Fig. 3(f)).

\section{Plasma tryptophan:large neutral amino acids and muscle fibre types}

In the LD-SDp groups, the experimental diets, but not photoperiod, significantly $(P=0.0099)$ affected plasma Trp:LNAA. A significant $(P=0 \cdot 0135)$ variation was detected among groups; post hoc analysis revealed that an $\alpha$-lactalbumin diet under SD significantly increased the ratio to the level in the LD control group (Fig. 4(a)). Trp:LNAA in the LDp-SD groups were affected by diet $(P=0.0027)$ and photoperiod $(P=0.0265)$ with significant variations among groups $(P=0.0038)$. Post boc analysis revealed that the Trp:LNAA in the SD control group were significantly $(P<0.05)$ lower than those in the LD control group. In contrast to the LD-SDp groups, ingestion of gluten and soya protein diets before exposure to SD significantly (gluten: $\quad P<0.01$, soya protein: $P<0.05)$ prevented the SD-induced suppression of Trp:LNAA (Fig. 4(b)). When correlation analysis was performed between Trp:LNAA and time spent in the centre area in the OFT, the values in the LDp-SD groups but not those in the LD-SDp groups were significantly correlated (LD-SDp; $P=0.2469, r$ 0.1952, LDp-SD; $P=0.0174$, $r$ 0.3996) (Fig. 4(c) and (d)). With regard to muscle fibre type, the SD control mice exhibited a higher percentage of IIb isoforms compared with the LD control mice $(P<0.0001$ in LD-SDp, $P<0.001$ in LDp-SD) (Fig. 4 (e) and (f)). However, in both the LD-SDp and LDp-SD groups, the experimental diets did not affect these ratios (Fig. 4(e) and (f)). 
(a)
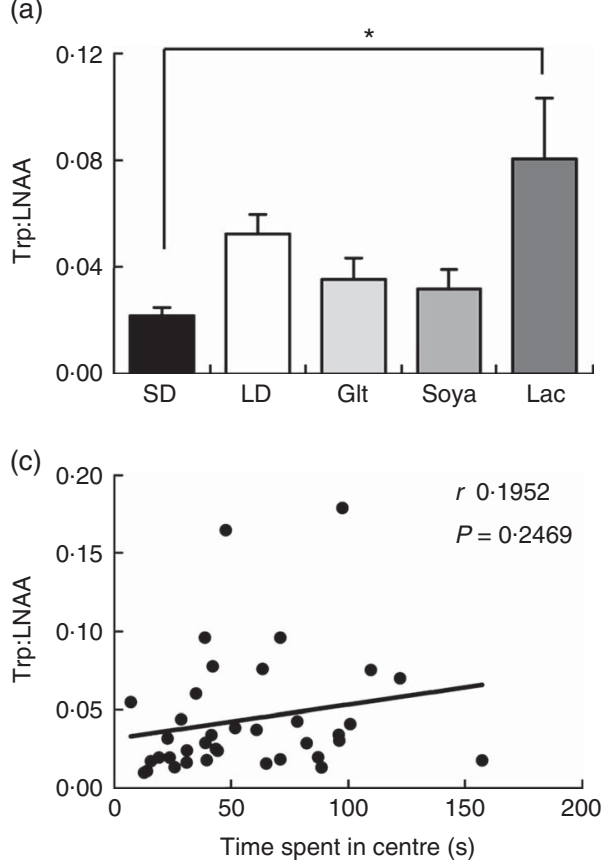

(e)

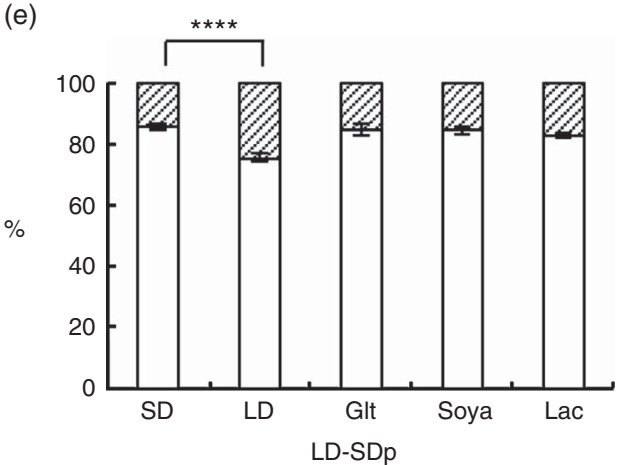

(b)
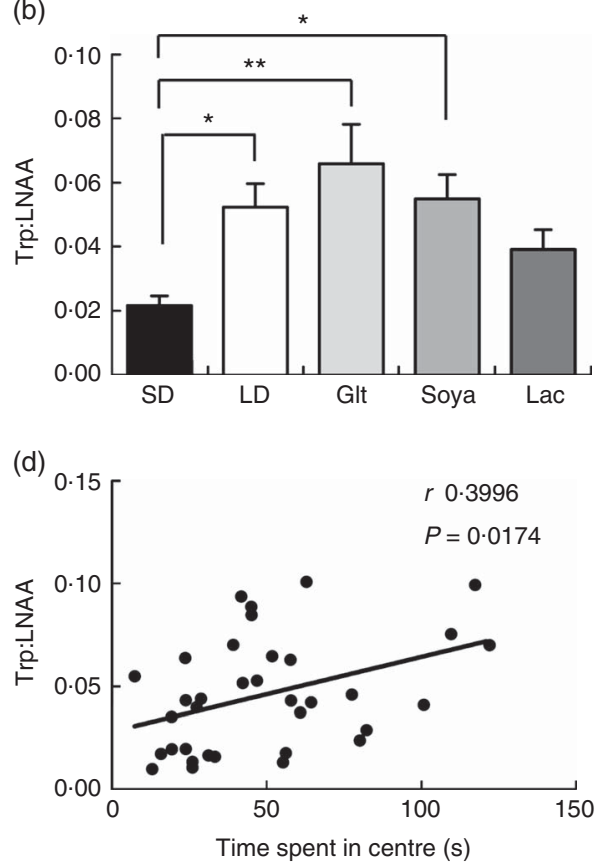

(f)

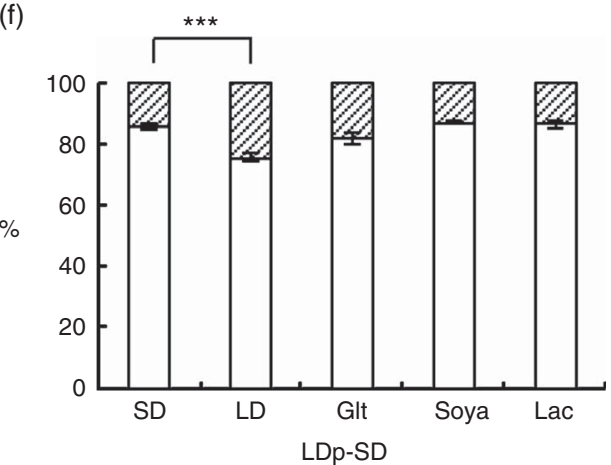

Fig. 4. Effects of various protein diets (glt, gluten; soya, soya protein; lac, $a$-lactalbumin) on plasma tryptophan:large neutral amino acids (Trp:LNAA) (a, b), correlation between plasma Trp:LNAA and time spent in the centre in the open field test (c, d), and ratio of muscle fibre type (e, f). Muscle fibre type is shown by the percentage of $\mathrm{Ilb}$ isoforms to the sum of difference: ${ }^{*} P<0.05$, ${ }^{\star *} P<0.01$, ${ }^{\star * *} P<0.001$, ${ }^{\star * * *} P<0.0001$ (Dunnett's test) $v$. SD control. $P$ and $\mathrm{r}$ values (c, d), correlation analysis, significance, $P<0.05$. LD, long-day condition; SD, short-day condition.

\section{Discussion}

In the OFT, SD control mice spent lesser time in the central area compared with LD control mice, which indicated anxiety-like behaviour. This is consistent with the findings of our previous study ${ }^{(5)}$, although the previous study showed photoperiodinduced anxiety-like behaviour using the elevated plus-maze test, but no effect was detected using the OFT. This discrepancy may be because of the differences in photoperiodic conditions; in the present study, LD and SD mice were housed under 18L:6D and 6L:18D, respectively, whereas in the previous study they were housed under 16L:8D and 8L:16D. The larger difference between SD and LD in the present study might enhance the photoperiod-induced anxiety-like behaviour. The present study further clarified that replacement of casein protein with $\alpha$-lactalbumin, which has a high Trp:LNAA, during SD significantly increased the time spent in the central area in the OFT (LD-SDp, Fig. 3(c)). This is consistent with the previous report that an $\alpha$-lactalbumin diet caused an anxiolytic-like effect in OFT or elevated plus-maze test under 12L:12D in C57BL/6J mice and rats ${ }^{(25,26)}$. These changes are unlikely to be a consequence of increased levels of spontaneous activity, because the number of lines crossed did not change with photoperiod. These results indicate that ingestion of $\alpha$-lactalbumin during seasons with short photoperiods, that is, fall and winter, can improve the anxiety-related problems that occur during these seasons ${ }^{(27,28)}$.

The mechanisms by which $\alpha$-lactalbumin regulates anxietylike behaviours remain unclear. A possible explanation is that high Trp:LNAA in the $\alpha$-lactalbumin diet enhance 5-HT signalling in the brain because of increased transport of plasma Trp through the blood-brain barrier, as plasma Trp:LNAA levels highly correlate with levels of brain $\operatorname{Trp}(r$ 0.95), and the sum of 5-HT and its major metabolite 5-hydroxyindoleacetic acid 
$(r 0.89)$, in rats ${ }^{(12)}$. In line with this hypothesis, chronic ingestion of $\alpha$-lactalbumin diet increased plasma Trp:LNAA in mice (this study) and rats ${ }^{(20)}$ and enhanced extracellular 5-HT levels in the rostromedial hypothalamus in rats ${ }^{(26)}$. However, there was no significant correlation between plasma Trp:LNAA levels and the time spent in the centre of the open field for the LD-SDp groups, although acute effects of dietary intake before the behavioural test and plasma collection might have affected the data. Compared with the other proteins used in this study, $\alpha$-lactalbumin contains a relatively high content of lysine, aspartate and branched-chain amino acids. These amino acids might also contribute to the observed behavioural changes. Notably, mice fed the $\alpha$-lactalbumin diet ate less food than LD control mice. It suggests that $\alpha$-lactalbumin diet did not induce LD-dependent biological processes. Metabolic changes as a consequence of low energy intake may be related to altered anxiety-like behaviour.

Our study demonstrated that the intake of a gluten diet before the transition to SD prevented the SD-induced increase in the time spent in the central area in the OFT (LDp-SD, Fig. 3(d)). Furthermore, mice on a gluten diet before SD exhibited high Trp:LNAA in plasma (Fig. 4(b)), which correlated with less anxiety-like behaviour. These effects of gluten were not regulated directly by the Trp:LNAA in gluten, as intake of gluten during SD did not have this effect, and the levels of Trp:LNAA in gluten were relatively low compared with that of Trp:LNAA in the other experimental diets. In addition, plasma Trp:LNAA in the LDp-SD group were not affected by time of day that affects eating behaviours and activity levels. These observations suggest that previously ingested gluten diet modulate basal levels of metabolism or absorption of amino acids. Notably, mice that were on a gluten diet ate less food during the period they were fed a gluten diet, even though food intake was restored after switching to a casein diet. Energy restriction is known to enhance insulin sensitivity in the muscles ${ }^{(29)}$, which consequently raises plasma Trp:LNAA through promotion of muscle uptake of several LNAA such as valine, leucine and isoleucine $^{(30)}$. This may explain the high Trp:LNAA levels in the mice fed a gluten diet before SD; these mice ate less and therefore had higher levels of Trp:LNAA. However, limited food intake cannot fully explain the OFT behaviour results, because the mice fed soya protein or an $\alpha$-lactalbumin diet in the LDp-SD group also had reduced food intake, although the OFT behaviour was unchanged. An additional mechanism might be related to the effect of gluten on Trp metabolism in the small intestine. Dietary gluten causes increased enzymatic activity of indoleamine 2,3-dioxygenase, a Trp catabolic enzyme, by autoimmune-mediated inflammation in celiac disease, which is caused by a dysregulated immune response towards gluten ${ }^{(31,32)}$. Dietary gluten also modifies gut microbiota ${ }^{(33)}$, which regulate absorption of amino acids, as well as enzymatic activity of coenzymes, for amino acid metabolism ${ }^{(34,35)}$. Generally, alteration of inflammation-related responses and microbiota occurs chronically after a latent period ${ }^{(33,36)}$. These delayed effects might be related to plasma Trp:LNAA and anxiety-like behaviour in the mice on a gluten diet.

In the FST, immobility in SD control mice was slightly $(P=0.073)$ lower than that in LD control mice. Ingestion of soya protein before the transition from LD to SD suppressed the immobility during SD compared with the SD control mice (LDp-SD, Fig. 3(f)). Soya protein intake was lower, similar to what was observed with gluten protein intake, and the link between limited energy and subsequent increase of Trp:LNAA may partially explain the results. However, other factors regulating FST behaviour are possible, because the results in the FST were highly different from the results of the OFT, in which $\alpha$-lactalbumin and gluten in the LD-SDp and LDp-SD groups, respectively, were effective in regulating the time spent in the central area. Anxiety- and depression-like behaviours are controlled by a distinct pathway. 5-HT receptor 2A-deficient mice exhibited more anxiety-like behaviours compared with wild-type mice, whereas no difference was observed in depression-like behaviours ${ }^{(37)}$. In contrast, the mice deficient in Trp hydroxylase (TPH)2, a rate-limiting enzyme in 5-HT synthesis, demonstrated modulation of depression-like, but not anxiety-like, behaviours ${ }^{(38)}$. These reports suggest that the synthetic pathway of 5-HT is specifically involved in regulation of depression-like behaviours. Although there is evidence that soya phyto-oestrogen induces TPH protein content in the brains of primates ${ }^{(39)}$, our data cannot be explained by a direct effect of soya protein, given that the immobility in the FST in the mice in LD-SDp was not modified by soya protein diet. Considering that energy intake was limited in the mice fed soya protein in the LDp-SD group, and that insulin sensitivity is enhanced by energy restriction ${ }^{(29)}$, enhanced action of insulin after the switch to casein might enhance the brain 5-HT signals. In support of this hypothesis, insulin stimulates TPH2 expression in the brains of chickens ${ }^{(40)}$. Alternately, phyto-oestrogen in soya protein diet under LD might induce long-term effect of oestrogen-related processes involved in regulation of aggression and anxiety ${ }^{(41)}$, given that sensitivity to oestrogen is regulated by photoperiod in mice ${ }^{(42)}$, and early exposure to oestradiol alters long-term behavioural sensitivity to anxiolytic drugs in rats ${ }^{(43)}$.

In our study, body and epididymal fat weights, as well as NEFA levels in plasma, were higher in the SD control group than in the LD control group, whereas food intake was not different between them. Previous studies that used Siberian hamsters or Fischer 344 rats, which are both long-day breeders, reported that body and epididymal fat weights were higher under LD than $\mathrm{SD}^{(44-46)}$. In contrast, Syrian hamsters show higher body weight under SD than $\mathrm{LD}^{(47)}$. Similarly, in humans, body weight increases from fall to winter ${ }^{(48)}$. These reports indicate that the direction of the photoperiodic response of body weight and fat mass differs between species. The present study further showed the photoperiodic changes in the ratios of muscle fibre types in $\mathrm{C} 57 \mathrm{BL} / 6 \mathrm{~J}$ mice; ratio of IIb isoforms, which represent fast-twitch fibres, to the sum of IIa and IIx isoforms, which represent intermediate types of fast- and slowtwitch muscle fibres, are lower under LD than SD. These changes in muscle fibre type might be related to photoperiodic changes in fat metabolism. Type IIa fibres have a higher capacity of oxidative metabolism than type IIb fibres ${ }^{(49)}$. High IIa/x fibre types in the mice under LD might be related to mobilisation of fatty acids through lipolysis of adipose tissue; released free fatty acids might be transported to muscle for further oxidation. This hypothesis is in line with the report that 
tribbles bomolog 3 overexpression, which promotes fatty acid oxidation, causes shift of muscle fibre types from fast- to slow-twitch fibres ${ }^{(50)}$, and can reasonably explain low mass of epididymal fat and plasma NEFA levels observed in our study.

The mice on an $\alpha$-lactalbumin diet during SD exhibited lower body and epididymal fat weights compared with the SD control mice. This is probably because of the reduced intake of $\alpha$-lactalbumin diet. This is consistent with the LDp-SD group results in which the mice on an $\alpha$-lactalbumin diet before the transition to SD exhibited suppressed food intake during the period in which they were fed an $\alpha$-lactalbumin diet, as well as reduced body and epididymal fat weight, and reduced NEFA levels as a consequence. Notably, the mice in the LDp-SD group consumed an equivalent amount of the casein diet after the transition to SD, suggesting that the reduction of body and epididymal fat weights was not a consequence of reduced food intake. Our study also showed that composition of muscle fibre type was unmodified by an $\alpha$-lactalbumin diet, indicating that the effect of this diet on fat and body weights is unrelated to muscle fibre type. Consistent with the findings of our study, several other studies reported that intake of $\alpha$-lactalbumin resulted in reduction of body and epididymal fat weights in C57BL/6J mice ${ }^{(25,51)}$.

In conclusion, our study demonstrated that various dietary proteins ingested before and during SD regulate anxiety- and depression-like behaviours, plasma Trp:LNAA, body weight and fat mass. Ingestion of appropriate proteins during specific seasons may be useful to improve or prevent winter-induced mood and metabolism problems, including symptoms of SAD.

\section{Acknowledgements}

The authors thank Nihon Shokuhin Kako Co., Ltd for providing the maize starch, Fuji Oil Co., Ltd for providing the soya protein and Meiji Co., Ltd for providing $\alpha$-lactalbumin.

This study was supported by Grants-in-Aid for JSPS Fellows to T. O. (no. 25.4372), Grants-in-Aid for Challenging Exploratory Research to S. Y. (no. 15K12341) from the Japanese Society for the Promotion of Science and a research grant from Urakami Foundation to S. Y.

S.Y. designed the study. T. O., R. G., A. I., M. K. and S. S. conducted the animal experiment. Y. O. and W. M. performed the analysis of muscle fibre type. S. Y., T. O. and M. F. analysed the data. S. Y. and T. O. wrote the paper. All authors discussed the results and commented on the manuscript.

The authors declare that they have no conflicts of interest.

\section{References}

1. Einat H, Kronfeld-Schor N \& Eilam D (2006) Sand rats see the light: short photoperiod induces a depression-like response in a diurnal rodent. Behav Brain Res 173, 153-157.

2. Lincoln GA (2002) Neuroendocrine regulation of seasonal gonadotrophin and prolactin rhythms: lessons from the Soay ram model. Reprod Suppl 59, 131-147.

3. Leach G, Adidharma W \& Yan L (2013) Depression-like responses induced by daytime light deficiency in the diurnal grass rat (Arvicanthis niloticus). PLOS ONE 8, e57115.
4. Otsuka T, Goto M, Kawai M, et al. (2012) Photoperiod regulates corticosterone rhythms by altered adrenal sensitivity via melatonin-independent mechanisms in Fischer 344 rats and C57BL/6J mice. PLOS ONE 7, e39090.

5. Otsuka T, Kawai M, Togo Y, et al. (2014) Photoperiodic responses of depression-like behavior, the brain serotonergic system, and peripheral metabolism in laboratory mice. Psychoneuroendocrinology 40, 37-47.

6. Pyter LM \& Nelson RJ (2006) Enduring effects of photoperiod on affective behaviors in Siberian hamsters (Phodopus sungorus). Behav Neurosci 120, 125-134.

7. Rosenthal NE, Sack DA, Gillin JC, et al. (1984) Seasonal affective disorder. A description of the syndrome and preliminary findings with light therapy. Arch Gen Psychiatry 41, 72-80.

8. Carlsson A, Svennerholm L \& Winblad B (1980) Seasonal and circadian monoamine variations in human brains examined post mortem. Acta Psychiatr Scand 280, 75-85.

9. Lambert GW, Reid C, Kaye DM, et al. (2002) Effect of sunlight and season on serotonin turnover in the brain. Lancet $\mathbf{3 6 0}$, $1840-1842$.

10. Maes M, Scharpe S, Verkerk R, et al. (1995) Seasonal variation in plasma L-tryptophan availability in healthy volunteers. Relationships to violent suicide occurrence. Arch Gen Psychiatry 52, 937-946.

11. Willeit M, Sitte HH, Thierry N, et al. (2008) Enhanced serotonin transporter function during depression in seasonal affective disorder. Neuropsychopharmacology 33, 1503-1513.

12. Fernstrom JD \& Wurtman RJ (1972) Brain serotonin content: physiological regulation by plasma neutral amino acids. Science 178, 414-416.

13. Hawkins RA, O'Kane RL, Simpson IA, et al. (2006) Structure of the blood-brain barrier and its role in the transport of amino acids. J Nur 136, 218S-226S.

14. Fernstrom JD \& Wurtman RJ (1971) Brain serotonin content: increase following ingestion of carbohydrate diet. Science 174, 1023-1025.

15. Lyons PM \& Truswell AS (1988) Serotonin precursor influenced by type of carbohydrate meal in healthy adults. Am J Clin Nutr 47, 433-439.

16. Rosenthal NE, Genhart MJ, Caballero B, et al. (1989) Psychobiological effects of carbohydrate- and protein-rich meals in patients with seasonal affective disorder and normal controls. Biol Psychiatry 25, 1029-1040.

17. Lam RW, Bowering TA, Tam EM, et al. (2000) Effects of rapid tryptophan depletion in patients with seasonal affective disorder in natural summer remission. Psychol Med 30, 79-87.

18. Choi S, Disilvio B, Fernstrom MH, et al. (2009) Meal ingestion, amino acids and brain neurotransmitters: effects of dietary protein source on serotonin and catecholamine synthesis rates. Physiol Behav 98, 156-162.

19. Choi S, DiSilvio B, Fernstrom MH, et al. (2011) The chronic ingestion of diets containing different proteins produces marked variations in brain tryptophan levels and serotonin synthesis in the rat. Neurochem Res 36, 559-565.

20. Feurte S, Gerozissis K, Regnault A, et al. (2001) Plasma Trp/ LNAA ratio increases during chronic ingestion of an alphalactalbumin diet in rats. Nutr Neurosci 4, 413-418.

21. Spangenburg EE \& Booth FW (2003) Molecular regulation of individual skeletal muscle fibre types. Acta Physiol Scand 178, 413-424.

22. Porsolt RD, Bertin A \& Jalfre M (1977) Behavioral despair in mice: a primary screening test for antidepressants. Arch Int Pharmacodyn Ther 229, 327-336.

23. Rubio LA (2003) Determination of diaminopimelic acid in rat feces by high-performance liquid chromatography using the 
Pico Tag method. J Chromatogr B Analyt Technol Biomed Life Sci 784, 125-129.

24. Mizunoya W, Wakamatsu J, Tatsumi R, et al. (2008) Protocol for high-resolution separation of rodent myosin heavy chain isoforms in a mini-gel electrophoresis system. Anal Biochem 377, 111-113.

25. Vekovischeva OY, Peuhkuri K, Backstrom P, et al. (2013) The effects of native whey and alpha-lactalbumin on the social and individual behaviour of C57BL/6J mice. Br I Nutr $\mathbf{1 1 0}$, $1336-1346$.

26. Orosco M, Rouch C, Beslot F, et al. (2004) Alpha-lactalbuminenriched diets enhance serotonin release and induce anxiolytic and rewarding effects in the rat. Behav Brain Res 148, $1-10$.

27. Graaf RD, Dorsselaer SV, Have MT, et al. (2005) Seasonal variations in mental disorders in the general population of a country with a maritime climate: findings from the Netherlands mental health survey and incidence study. $A m$ J Epidemiol 162, 654-661.

28. Schlager D, Schwartz JE \& Bromet EJ (1993) Seasonal variations of current symptoms in a healthy population. $\mathrm{Br} J$ Psychiatry 163, 322-326.

29. Dean DJ, Brozinick JT Jr, Cushman SW, et al. (1998) Calorie restriction increases cell surface GLUT-4 in insulin-stimulated skeletal muscle. Am J Physiol 275, E957-E964.

30. Lu J, Xie G, Jia W, et al. (2013) Insulin resistance and the metabolism of branched-chain amino acids. Front Med $\mathbf{7}$, 53-59.

31. Ciccocioppo R, Camarca A, Cangemi GC, et al. (2014) Tolerogenic effect of mesenchymal stromal cells on gliadinspecific $\mathrm{T}$ lymphocytes in celiac disease. Cytotherapy 16, 1080-1091.

32. Sollid LM (2002) Coeliac disease: dissecting a complex inflammatory disorder. Nat Rev Immunol 2, 647-655.

33. Bengmark S (2013) Gut microbiota, immune development and function. Pharmacol Res 69, 87-113.

34. Booth CC \& Brain MC (1962) The absorption of tritiumlabelled pyridoxine hydrochloride in the rat. J Physiol 164, 282-294.

35. Coburn SP, Mahuren JD, Wostmann BS, et al. (1989) Role of intestinal microflora in the metabolism of vitamin $\mathrm{B}_{6}$ and 4 '-deoxypyridoxine examined using germfree guinea pigs and rats. J Nutr 119, 181-188.

36. Yoshida R, Urade $\mathrm{Y}$, Tokuda $\mathrm{M}$, et al. (1979) Induction of indoleamine 2,3-dioxygenase in mouse lung during virus infection. Proc Natl Acad Sci U S A 76, 4084-4086.

37. Weisstaub NV, Zhou M, Lira A, et al. (2006) Cortical 5-HT2A receptor signaling modulates anxiety-like behaviors in mice. Science 313, 536-540.
38. Mosienko V, Bert B, Beis D, et al. (2012) Exaggerated aggression and decreased anxiety in mice deficient in brain serotonin. Transl Psychiatry 2, e122.

39. Shively CA, Mirkes SJ, Lu NZ, et al. (2003) Soy and social stress affect serotonin neurotransmission in primates. Pharmacogenomics J 3, 114-121.

40. Rice BB, Zhang W, Bai S, et al. (2014) Insulin-induced hypoglycemia associations with gene expression changes in liver and hypothalamus of chickens from lines selected for low or high body weight. Gen Comp Endocrinol 208, 1-4.

41. Spiteri T, Musatov S, Ogawa S, et al. (2010) The role of the estrogen receptor alpha in the medial amygdala and ventromedial nucleus of the hypothalamus in social recognition, anxiety and aggression. Behav Brain Res 210, 211-220.

42. Trainor BC, Rowland MR \& Nelson RJ (2007) Photoperiod affects estrogen receptor alpha, estrogen receptor beta and aggressive behavior. Eur J Neurosci 26, 207-218.

43. Calza A, Sogliano C, Santoru F, et al. (2010) Neonatal exposure to estradiol in rats influences neuroactive steroid concentrations, GABAA receptor expression, and behavioral sensitivity to anxiolytic drugs. J Neurochem 113, 1285-1295.

44. Bartness TJ (1996) Photoperiod, sex, gonadal steroids, and housing density affect body fat in hamsters. Physiol Behav $\mathbf{6 0}$, 517-529.

45. Heideman PD \& Sylvester CJ (1997) Reproductive photoresponsiveness in unmanipulated male Fischer 344 laboratory rats. Biol Reprod 57, 134-138.

46. Togo Y, Otsuka T, Goto M, et al. (2012) Photoperiod regulates dietary preferences and energy metabolism in young developing Fischer 344 rats but not in same-age Wistar rats. Am J Physiol Endocrinol Metab 303, E777-E786.

47. McElroy JF \& Wade GN (1986) Short photoperiod stimulates brown adipose tissue growth and thermogenesis but not norepinephrine turnover in Syrian hamsters. Physiol Behav 37, 307-311.

48. Ma Y, Olendzki BC, Li W, et al. (2006) Seasonal variation in food intake, physical activity, and body weight in a predominantly overweight population. Eur J Clin Nutr 60, 519-528.

49. Wessel TV, Haan AD, WJVD Laarse, et al. (2010) The muscle fiber type-fiber size paradox: hypertrophy or oxidative metabolism? Eur J Appl Physiol 110, 665-694.

50. An D, Lessard SJ, Toyoda T, et al. (2014) Overexpression of TRB3 in muscle alters muscle fiber type and improves exercise capacity in mice. Am J Physiol Regul Integr Comp Physiol 306, R925-R933.

51. Shi J, Lehmus AA, Pilvi TK, et al. (2011) Comparison of the metabolic effects of milk-derived $\alpha$-lactalbumin and amino acids mixture with equal composition in diet-induced obese mice. J Funct Foods 3, 70-78. 